\title{
Sistemas Emergentes no Ecossistema Digital Brasileiro de Saúde Pública: Uma Abordagem Sociotécnica
}

\author{
Sandro Luís Freire de Castro Silva ${ }^{1}$, Marcelo Fornazin ${ }^{1,2}$, \\ Rodrigo Pereira dos Santos ${ }^{1}$ \\ ${ }^{1}$ Programa de Pós-Graduação em Informática (PPGI) \\ Universidade Federal do Estado do Rio de Janeiro (UNIRIO) \\ Avenida Pasteur, 458 - Urca - Rio de Janeiro - RJ - Brasil \\ ${ }^{2}$ Instituto de Computação - Universidade Federal Fluminense (UFF) \\ Avenida General Milton Tavares de Souza, s/n - São Domingos - Niterói - RJ - Brasil \\ sandro.silva@uniriotec.br, fornazin@ic.uff.br, rps@uniriotec.br
}

\begin{abstract}
Brazilian National Health System has been treated as a priority in discussions of political agendas and still lives with precariousness. Solutions to related problems in most cases suggest the implementation of Health Information Systems. The question is such implementation, or any other underlying activity, should not be limited strictly to a technical perspective. The aim of this work is to investigate the emergent complexity of relationships in digital ecosystems, using the Brazilian Public Health Digital Ecosystem as a scenario in order to contribute with a sociotechnical view on the theme.
\end{abstract}

Resumo. O Sistema Único de Saúde convive com a precariedade mesmo sendo prioridade nas discussões das agendas políticas. As soluções para problemas relacionados na maioria dos casos sugerem a implantação de Sistemas de Informação em Saúde. A questão é que a implantação, ou qualquer outra atividade, não deve estar limitada ao olhar estritamente técnico. O objetivo desse trabalho de doutorado é descrever a complexidade emergente das relações em ecossistemas digitais, usando como cenário o Ecossistema Digital de Saúde Pública Brasileiro, a fim de contribuir com um olhar sociotécnico sobre o tema.

\section{Introdução}

A prestação de serviços de saúde pública no Brasil sempre foi tratada como prioridade nas discussões das agendas políticas. Contudo, o Sistema Único de Saúde (SUS) ainda convive com a precariedade [Saldiva e Veras 2018]. São inúmeros os fatores que contribuem com a ineficiência da assistência de saúde ao cidadão brasileiro. Entre os principais fatores, estão: o subfinanciamento frente aos desafios da saúde pública; a ausência de profissionais qualificados; má gestão dos recursos financeiros; e ausência de protocolos de atendimento eficientes [Mendes 2018].

As soluções para esses problemas passam por campos multidisciplinares, como a reformulação de processos ou até mesmo a revisão de práticas clínicas. No entanto, na maioria dos casos, as propostas sugerem a implantação de Sistemas de Informação em Saúde (SIS). A questão é que o trabalho de implantação de um SIS, ou qualquer outra 
atividade relacionada, não deve se limitar ao olhar estritamente técnico sobre o cenário, uma vez que é crescente a existência de estudos que corroboram com o fato de que a atividade requer uma análise mais detalhada dos atores, organizações governamentais, internacionais, órgãos de fomento e outros [Braa et al. 2017; Sengupta e Sahay 2018]. No contexto brasileiro, podem ser encontrados estudos que evidenciam a necessidade de observar esses aspectos quando se trata especificamente de SIS [Joia e Magalhães 2017].

Pode-se tomar o caso dos tratamentos oncológicos como meio de exemplificar essa perspectiva de análise. $\mathrm{O}$ processo de conclusão de um diagnóstico de câncer por meio do serviço brasileiro de saúde pública pode ser uma tarefa complexa, que exige relações entre diferentes profissionais e organizações de saúde, além de sistemas informacionais, digitais e analógicos.

Uma perspectiva para a tarefa de fornecer um diagnóstico é pensar que, com a dinamicidade do fluxo de informações, é possível que a discussão de casos seja realizada por meio de mensagens trocadas via smartphones [Giordano et al. 2017a]. Em um mundo no qual as pessoas estão conectadas, não é surpresa que a comunidade médica possua grupos de mensagens formados na maioria dos casos por colegas de faculdade e de trabalho. Assim, os profissionais de saúde lançam mão de uma série de artefatos, por exemplo, exames e portais de periódicos científicos de medicina, os quais são agenciados com o intuito de "fechar um diagnóstico".

Outro caso em particular é a forma no qual as filas de atendimento são conduzidas. O modelo que ainda prevalece hegemônico no Brasil é aquele centrado no médico [Silveira et al. 2018] e confere a esse profissional a gestão e a responsabilidade pelo controle das filas de atendimento de seu serviço diário, mesmo em ambientes controlados pelo Sistema Nacional de Regulação (SISREG). Na ausência de um sistema de informação para realizar o controle dessas filas, é possível utilizar uma planilha ou bloco de anotações para realizar esse controle.

Os casos citados são exemplos do que pode ser caracterizado como sistema emergente, que podem ser definidos como qualquer artefato que não foi inicialmente projetado para a atividade, mas é apropriado pelos atores e utilizado para atingimento de um determinado objetivo [Johnson 2001]. No domínio da saúde, esses sistemas vêm cada vez mais se tornando fundamentais para o desenvolvimento da prática, tornandoos parte significativa de um determinado contexto, convivendo com a ausência de controles ou regulação e tornando esse tipo de sistema um artefato informal em meio aos aparatos considerados oficiais ou formais.

Dessa forma, este trabalho de doutorado tem como objetivo investigar e descrever a complexidade emergente das relações em ecossistemas digitais, usando como cenário o Ecossistema Digital de Saúde [Hadzic e Chang 2010] no contexto da saúde pública brasileira, partindo do pressuposto que esse cenário não possui processos estritamente lineares e que sua análise mais aprofundada pode vir a mostrar sua real complexidade.

Pode-se considerar que a presente pesquisa faz parte do grande desafio que engloba a Visão Sociotécnica de Sistemas de Informação, contido nos Grandes Desafios da área de Sistemas de Informação no Brasil [Boscarioli et al. 2017]. Além desta seção 
de introdução, este artigo está organizado da seguinte forma: a Seção 2 que apresenta o problema; logo após, na Seção 3, é apresentada a proposta de solução, na qual descrevemos o método de pesquisa utilizado; após isso, o projeto de trabalho é apresentado na Seção 4, sucedido das atividades realizadas na Seção 5; por fim, na Seção 6 são apresentadas as considerações finais.

\section{Apresentação do Problema}

Muitas vezes, os processos do domínio da saúde pública nacional são vistos como um fluxo, o que simplifica a sua complexidade. A generalização desses cenários oculta suas particularidades, fazendo com que a percepção sobre o ecossistema digital de saúde pública pareça algo simples [Marinho e Cardoso 2007; Silva et al. 2018].

Em muitos casos, as práticas de concepção e manutenção de Sistemas de Informação abdicam de uma visão pluridisciplinar, abrindo mão de observar seu comportamento emergente, a complexidade de suas redes e outros aspectos peculiares quando se trata de sistemas complexos [Júnior et al. 2018]. É importante compreender que o problema não está na representação dos detalhes ou na complexidade do processo - a questão é que os modelos não descrevem as ações informais, sobretudo a colaboração e os desvios no processo para garantir seu sucesso.

A complexidade do cenário do ecossistema digital brasileiro de saúde pública é pouco conhecida pelos profissionais e pesquisadores em Sistemas de Informação. Nesse sentido, os esforços desta pesquisa de doutorado estarão concentrados em produzir elementos para supressão dessa lacuna.

\section{Proposta de Solução}

O objetivo desse trabalho é investigar diferentes casos do ecossistema digital brasileiro de saúde pública, com enfoque na descrição sociotécnica do caso, levando em consideração as práticas de profissionais de saúde e artefatos técnicos, tais como os sistemas de informação e, principalmente, os sistemas emergentes. Para tanto, foi identificada uma bibliografia que discute SIS considerando o olhar sociotécnico [Braa et al. 2017; Manya et al. 2018], bem como o uso de sistemas emergentes como o WhatsApp e outros artefatos [Giordano et al. 2017; Pimmer et al. 2017].

Com base na bibliografia, o trabalho irá teorizar a respeito dos sistemas emergentes no contexto da saúde pública brasileira. Além disso, pretende-se exemplificar as oportunidades da aplicação de abordagens sociotécnicas para descrever os cenários estudados.

\section{Projeto de Avaliação da Solução}

Por considerar a abrangência das questões envolvidas no caso do ecossistema digital brasileiro de saúde pública e a necessidade de coletar dados peculiares ao campo estudado, a pesquisa tem natureza qualitativa [Richardson 2014]. Inicialmente, buscouse conduzir um estudo para descrever a complexidade envolvida no uso do WhatsApp para fornecimento de diagnósticos e comunicação entre profissionais da saúde. Além deste estudo, a pesquisa pretende investigar os sistemas emergentes considerados "não formais" no contexto da saúde em um estudo específico e, em seguida, se debruçar em descrever o acoplamento de sistemas emergentes a tecnologias já existentes, como 
videogames e artefatos do tipo, consistindo em um terceiro estudo. Em seguida, os três estudos serão analisados para teorizá-los por meio de uma triangulação das reflexões.

A abrangência e o escopo desta pesquisa estão limitados à realidade nacional e a casos específicos do estado do Rio de Janeiro. No entanto, acredita-se que, por se tratar de um sistema de saúde nacional, os processos e métodos não se diferenciam de outros estados e a qualidade na prestação do serviço deve ser desconsiderada para eventuais generalizações. Um mapeamento sistemático da literatura está sendo realizado para caracterizar sistemas emergentes neste tipo de ecossistema digital. A pesquisa bibliográfica terá seu embasamento principal em livros e artigos científicos que tangenciam os assuntos abordados [Bardin 2015].

A abordagem utilizada para o desenvolvimento dessa pesquisa pode ser caracterizada como interpretativa [Walsham 2006]. O trabalho realizará múltiplos estudos de caso em conjunto com a utilização da Teoria Ator-Rede, com o objetivo de analisar as relações dos profissionais saúde do Brasil com os sistemas de informação em saúde existentes. Os estudos de caso em SI, em sua maioria, adotam uma visão positivista [Paré 2018]. Nesse tipo de estudo recomenda-se reunir todo material ao alcance em um diário de bordo, a fim de se reconstruir a trajetória dos artefatos técnicos [Latour 1987]. O pesquisador que está conduzindo essa pesquisa de doutorado participa do cotidiano de trabalho da divisão de informática de um hospital brasileiro e pretende reunir as fontes de evidência por meio de: a) entrevistas; b) observações participante e não participante; e c) fontes documentais.

\section{Atividades Realizadas}

Um trabalho sobre o tema foi apresentado XI Simpósio Nacional da Associação Brasileira de Pesquisadores em Cibercultura, intitulado "o Ecossistema Digital de Diagnósticos Oncológicos Brasileiro: uma Análise do uso de Sistemas de Informação em pareceres médicos" [Silva et al. 2018]. O trabalho abordou um recorte do Ecossistema de Saúde Pública Brasileiro e teve como objetivo investigar um caso de elaboração de diagnósticos oncológicos na rede pública de saúde do estado do Rio de Janeiro.

Além disso, um mapeamento sistemático da última década do IEEE CBMS International Symposium on Computer-Based Medical Systems foi realizado com o objetivo de identificar sistemas emergentes no conjunto de artigos publicados neste evento de referência no assunto e foi aceito para publicação em 2019 [Silva et al. 2019]. Esse trabalho relevante para a presente pesquisa serviu de motivação para a realização de um mapeamento sistemático com maior abrangência, que está em andamento.

\section{Considerações Finais}

No estágio atual da pesquisa, pode-se concluir que os sistemas emergentes são visivelmente auto organizáveis, uma vez que a dinâmica de seu funcionamento sofre constantes modificações, por se tratar de um serviço público. A frequente mudança de atores e a forte influência na fronteira do ecossistema digital brasileiro de saúde pública o caracteriza como fortemente auto-organizável. 
A emergência desses sistemas se dá, em parte, pelo fato de não ser percebida a real complexidade na forma com que sistemas tradicionais "formais" são concebidos. A ausência dessa sensibilidade faz com que sistemas emergentes ditos "não formais" passem a fazer parte do cenário. Cabe a reflexão a respeito das práticas de concepção de sistemas de informação e até que ponto o olhar sociotécnico está sendo considerado.

\section{Referências}

Araujo, R. M., Maciel, R. S. e Boscarioli, C. (2017). I GranDSI-BR - Grandes Desafios de Pesquisa em Sistemas de Informação no Brasil 2016 a 2026 - Relatório Técnico.

Bardin, L. (2015). Análise de conteúdo (Edição revista e actualizada). Lisboa: Edições 70 ,

Braa, J., Sahay, S., Lewis, J. e Senyoni, W. (2017). Health information systems in Indonesia: Understanding and addressing complexity. In IFIP Advances in Information and Communication Technology.

Braa, Monteiro e Sahay (2017). Networks of Action: Sustainable Health Information Systems across Developing Countries. MIS Quarterly,

Bruno Silveira, C., Suelen Pontes Costa, L. e Bessa Jorge, M. S. (2018). Redes de atenção à saúde como produtoras de cuidado em saúde mental: uma análise reflexiva. Revista Portuguesa de Enfermagem de Saúde Mental, n. 19, p. 61-70.

Finkelstein, J. e Wood, J. (2011). Implementing home telemanagement of congestive heart failure using Xbox gaming platform. Proceedings of the Annual International Conference of the IEEE Engineering in Medicine and Biology Society, EMBS, p. 31583163.

Fornazin, M. e Joia, L. A. (2015). Remontando a Rede De Atores Na Implantação De Um Sistema De Informação Em Saúde. Revista de Administração de Empresas, v. 55, n. 5 , p. 527-538.

Ganasegeran, K., Renganathan, P., Rashid, A. e Al-Dubai, S. A. R. (2017). The mHealth revolution: Exploring perceived benefits of WhatsApp use in clinical practice. International Journal of Medical Informatics,

Giordano, V., Koch, H., Godoy-Santos, A., et al. (2017). WhatsApp Messenger as an Adjunctive Tool for Telemedicine: An Overview. Interactive Journal of Medical Research, v. 6, n. 2, p. e11.

Hadzic, M. e Chang, E. (2010). Application of digital ecosystem design methodology within the health domain. IEEE Transactions on Systems, Man, and Cybernetics Part A:Systems and Humans, v. 40, n. 4, p. 779-788.

Johnson, S. (2001). Emergence: The Connected Lives of Ants, Brains, Cities, and Software. IEEE Spectrum

Júnior, B. H. P., Maciel, M. E. de S. S., Bonfim, W. S., et al. (2018). Desenvolvimento de um software para suporte à avaliação fisioterápica baseado na Classificação Internacional de Funcionalidade, Incapacidade e Saúde.

Latour, B. (1987). Science in Action. 
Manya, A., Braa, J. e Sahay, S. (2015). A Socio-Technical Approach to Understanding Data Quality in Health Information Systems: Data Quality Intervention in Kenya. ISTAfrica 2015 Conference Proceedings,

Manya, A., Sahay, S., Braa, J. e Shisia, B. (2018). Understanding the Effects of Decentralization on Health Information Systems in Developing Countries: A Case of Devolution in Kenya. In IST-Africa 2018 Conference Proceedings Paul Cunningham and Miriam Cunningham (Eds) IIMC International Information Management Corporation, 2018.

Marinho, A. e Cardoso, S. de S. (2007). Um estudo multinível sobre as filas para internações relacionadas com a gravidez, o parto e o puerpério no SUS. Economia Aplicada, v. 11, n. 4, p. 527-554.

Mendes, E. V. (2018). Entrevista: A abordagem das condições crônicas pelo Sistema Único de Saúde. Ciência \& Saúde Coletiva, v. 23, n. 2, p. 431-436.

Nikolic, A., Wickramasinghe, N., Claydon-Platt, D., Balakrishnan, V. e Smart, P. (2018). The use of communication apps by medical staff in the australian health care system: Survey study on prevalence and use. Journal of Medical Internet Research

Paré, G. (2018). Investigating Information Systems with Positivist Case Research. Communications of the Association for Information Systems, v. 13, n. February.

Pimmer, C., Mhango, S., Mzumara, A. e Mbvundula, F. (2017). Mobile instant messaging for rural community health workers: A case from Malawi. Global Health Action,

Richardson, R. J. (2014). Pesquisa social: métodos e técnicas.

Saldiva, P. H. N. e Veras, M. (2018). Gastos públicos com saúde: breve histórico, situação atual e perspectivas futuras. Estudos Avançados, v. 32, n. 92, p. 47-61.

Sengupta, S. e Sahay, A. (2018). Social enterprises in the Indian context: conceptualizing through qualitative lens. Journal of Global Entrepreneurship Research,

Silva, S. L. F. C., Antonio, N. P., Fornazin, M. e Santos, R. P. Dos (2019). Looking for Emergent Systems in Computer-Based Medical Systems: A Review from the Last Decade. In: CBMS International Symposium on Computer-Based Medical Systems. In Proceedings of the 32th IEEE CBMS.

Silva, S. L. F. C., Fornazin, M., Araujo, R. M. e Dos Santos, R. P. (2018). O Ecossistema Digital de Diagnósticos Oncológicos Brasileiro: Uma Análise do Uso de Sistemas de Informação em Pareceres Médicos. In XI Simpósio Nacional da ABciber.

Silva, R. K. e, Moraes, E. M., Mota, N. V. y V. P. Da e Mourad, R. G. (2018). Simulação e teoria das filas aplicadas na análise dos encaminhamentos de pacientes SUS para a especialidade médica de angiologia. Revista de Administração em Saúde, v. 18, n. 72 .

Walsham, G. (2006). Doing interpretive research. European Journal of Information Systems, v. 15, n. 3, p. 320-330. 